Review began 02/24/2022 Review ended 02/26/2022 Published 03/01/2022

() Copyright 2022

Andan et al. This is an open access article distributed under the terms of the Creative Commons Attribution License CC-BY 4.0. which permits unrestricted use, distribution, and reproduction in any medium, provided the original author and source are credited.

\section{Evaluation of the Reliability and Quality of YouTube Videos on Ovarian Cysts}

\author{
Cengiz Andan ${ }^{1}$, Mustafa F. Aydin ${ }^{1}$ \\ Research Hospital, Diyarbakir, TUR \\ Corresponding author: Cengiz Andan, mdcengizandan@gmail.com
}

1. Obstetrics and Gynecology, TC Ministry of Health, Health Sciences University Gazi Yasargil Diyarbakır Training and

\section{Abstract}

\section{Aim}

The aim of this study is to investigate the quality and reliability of YouTube videos containing content related to ovarian cysts.

\section{Methods}

The search terms “Ovarian Cyst”, “Ovarian Cyst Symptoms”, “Ovarian Cyst Treatment”, and “Ovarian Cyst Diagnosis” were searched on YouTube. A total of 110 videos were examined and repetitive videos, nonEnglish videos, videos with advertising content, videos with entertainment and news content, and videos with very poor image and sound quality were excluded. Finally, the videos included in the study were evaluated using DISCERN and Global Quality Scale (GQS).

\section{Results}

It was found that 50 videos examined in this study were uploaded between the years 2014 and 2020, with an average of $492.252 \pm 710.768$ and a total of 24.612 .595 views. The mean DISCERN score given to 50 videos analyzed by two researchers was $2.81 \pm 1.3$ and the mean GQS score was $2.88 \pm 1.4$. When we divided the scores given to the videos by two researchers into three groups, it was determined that 27 ( $54 \%$ ) of the 50 videos were in the misleading/poor quality group, nine (18\%) were in the medium quality group, and 14 (28\%) were in the useful/quality group.

\section{Conclusion}

It has been determined that the videos with "ovarian cyst" content on YouTube are generally of poor quality. Bad quality videos were uploaded by non-doctors and attracted more attention than videos uploaded by doctors.

Categories: Obstetrics/Gynecology

Keywords: gqs, discern, internet, youtube, ovarian cyst

\section{Introduction}

An ovarian cyst is a sac filled with fluid or semi-fluid material that arises in one of the ovaries. The number of diagnosed ovarian cysts has increased due to regular examinations and advances in ultrasound technology [1]. Although most patients with ovarian cysts are asymptomatic, some cysts are associated with a range of symptoms, and those that are malignant often do not cause symptoms until the advanced stage [2].

Although ovarian cysts are frequently seen in women between the ages of 20 and 45, they can occur at any age from the neonatal period to the post-menopausal period. These cysts are usually detected incidentally during an ultrasound or pelvic examination. These cysts are usually small in size and self-limiting [3]. However, it is known that the detection of ovarian cysts causes anxiety in women [4]. These patients often turn to other resources for finding solutions and sharing experiences and worries before seeking medical help.

In studies, it is reported that patients and their relatives primarily search for information about their health on the Internet and do not share this information with their doctors [5-6]. At the top of these websites are platforms such as Google (www.google.com) and YouTube (www.youtube.com). These platforms contain very educational and useful information, as well as highly misleading and harmful information. However, the usefulness of this information cannot be distinguished by the patients and their relatives. YouTube is the world's largest video-sharing website and is visited by millions of users daily. According to YouTube statistics, there are over 2 billion YouTubers globally, which corresponds to approximately one-third of all Internet users on a global scale. In addition, considering that approximately one billion hours of views are 
made per day, it is clear how widely used YouTube is around the world [7]. In many studies evaluating the quality of health-related YouTube videos, it has been reported that the quality of the videos examined, especially those uploaded by non-doctor users, is generally poor or moderate [8-14]. However, in our literature review, we did not find a study in which ovarian cyst-containing videos on YouTube were examined. The aim of this study is to investigate the quality and reliability of ovarian cyst content videos on YouTube.

\section{Materials And Methods}

\section{Ethical disclosure}

Since only public-access data was used in this study, it was exempted from ethics committee approval.

\section{Study design}

This study was designed by two gynecologists who are experts in their field on 01/06/2021. The search terms to be used for the research were determined using the Google Trends (https://rends.google.com) application. A search was made by entering the term "Ovarian Cyst" in the Google Trends application and selecting "Entire World" and the last five years from the filters. In our study, the search terms "Ovarian Cyst", “Ovarian Cyst Symptoms”, “Ovarian Cyst Treatment”, and “Ovarian Cyst Diagnosis” were used with both the results of the Google Trends application and the consensus of the two researchers. In addition, a Microsoft Excel file (Microsoft Corporation, Redmond, WA) was prepared by the researchers in order to save the data. In this Excel file, the video link, uploader's quality, video's content, length (minutes), total number of views, date it was uploaded, date it was viewed, time since the video was uploaded, number of comments, likes, and dislikes, and Video Power Indexes (VPI) have been recorded. Finally, the study design was created by deleting the past searches and cookies of the computer on which the research was to be conducted.

\section{Data collection}

Two researchers searched for the determined search terms and used the "relevance" and "view counts" filters. For each search term, the most relevant and most viewed videos on the subject were examined and the information of all the videos included in the study was saved in an Excel file. In the study, a total of 110 videos were examined and repetitive videos, non-English videos, videos with advertising content, videos with entertainment and news content, videos with very poor image and sound quality were excluded. The 50 most relevant and most viewed videos that fit the study sample were included in the study.

\section{Evaluation of the data}

The 50 videos included in the study were evaluated by the researchers in separate environments using the Quality Criteria for Consumer Health Information (DISCERN) [15] and Global Quality Scale (GQS) [16], which were previously used in many YouTube studies [8-9,12,14]. The structured DISCERN scale is used to determine the reliability of videos. The scale consists of five questions in total, showing that 1-2 points are bad, 3 points are medium, 4 points are good, and 5 points are perfectly reliable. Similarly, the GQS scale consists of five questions and shows the quality of the videos. The questions of the DISCERN and GQS scale are given in Table 1 . 


\section{Cureus}

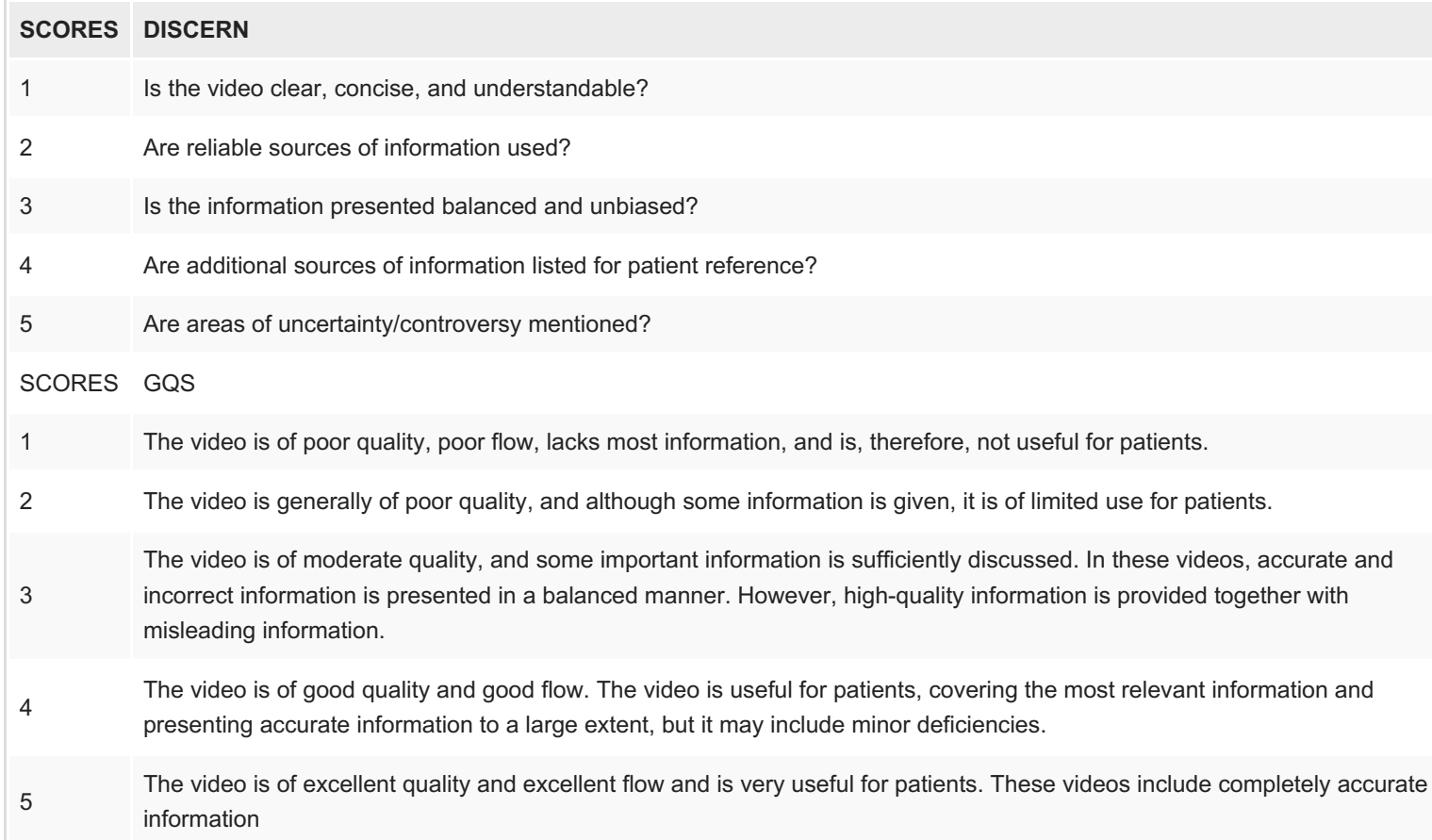

\section{TABLE 1: Questions related to DISCERN and GQS}

According to the scores given to the videos by the two researchers, videos with one to two points were classified as misleading/harmful videos, videos with three points as medium quality/safe videos, and videos with four to five points as useful/quality videos.

\section{Statistical analysis}

The data obtained in the study were statistically analyzed using the Statistical Package for the Social Sciences version 20.0 software (IBM Corp., Armonk, NY). Descriptive data are expressed as numbers, percentages, and median (minimum-maximum) values. The conformity of the data to the normal distribution was analyzed with the Shapiro-Wilk test. The agreement between the two researcher physicians was evaluated using the Kappa coefficient. A p-value of $<0.05$ was considered statistically significant.

\section{Results}

It was found that 50 videos examined in this study were uploaded between the years 2014 and 2020, with an average of $492.252 \pm 710.768$ and a total of 24.612 .595 views. It was determined that the average number of views per day was $506.82 \pm 601$. It was also determined that eight (16\%) of the videos were animated and 42 of them were real images. The data on the general characteristics of the videos are given in Table 2. 


\section{Cureus}

\begin{tabular}{|c|c|c|c|c|c|c|}
\hline & n (\%) & n (\%) & View & Comment & Like & Dislike \\
\hline \multicolumn{7}{|l|}{ Image } \\
\hline Real & $8(16)$ & 19.649 .132 & 1.725 & 39.589 & 1.208 & $92.60 \pm 3.3$ \\
\hline Animation & $42(74)$ & 16.274 .401 & 20.392 & 198.184 & 8.396 & $94.10 \pm 4.8$ \\
\hline \multicolumn{7}{|l|}{ Uploaders } \\
\hline Physician & $15(30)$ & 15.443 .085 & 10.148 & 18.440 & 6773 & $94.34 \pm 5.4$ \\
\hline Hospital Channel & $4(8)$ & 985.324 & 558 & 4.071 & 250 & $94.46 \pm 1.2$ \\
\hline Health Channel & $5(10)$ & 2.412.236 & 3.757 & 13.609 & 1.076 & $94.17 \pm 3.7$ \\
\hline Patients & $13(26)$ & 401.057 & 3.005 & 5.009 & 263 & $93.27 \pm 5.4$ \\
\hline Herbalist & $2(4)$ & 2.546 .883 & 6.181 & 43.500 & 2.470 & $88.12 \pm 7.3$ \\
\hline Blog Channel & $5(10)$ & 9.402 .046 & 4.875 & 99.100 & 4.973 & $94.53 \pm 3.3$ \\
\hline Yoga Channel & $6(12)$ & 4.718 .912 & 5.667 & 73.000 & 2.261 & $94.84 \pm 4.6$ \\
\hline \multicolumn{7}{|l|}{ Content } \\
\hline General Info & $10(20)$ & 2.752 .411 & 2.088 & 12.653 & 871 & $93.34 \pm 3.2$ \\
\hline Surgical Technique & $7(14)$ & 1.712 .328 & 931 & 8.021 & 681 & $93.38 \pm 4.2$ \\
\hline Patient Experience & $14(28)$ & 407.312 & 3.008 & 5.022 & 263 & $93.77 \pm 5.4$ \\
\hline Yoga & $5(10)$ & 3.926 .829 & 4.164 & 69.600 & 1.697 & $95.54 \pm 4.6$ \\
\hline Herbal & $9(18)$ & 10.371 .599 & 15.079 & 147.800 & 7.311 & $93.5 \pm 4.6$ \\
\hline Symptom & $2(4)$ & 14.791 .616 & 7.467 & 259.000 & 6.100 & $96.76 \pm 1.2$ \\
\hline Diet & $3(6)$ & 1.948 .448 & 1.456 & 12.800 & 1.143 & $93.54 \pm 2.7$ \\
\hline
\end{tabular}

TABLE 2: Data on the general characteristics of videos

It was determined that the average length of the 50 videos examined was $8.25 \pm 6.1$ minutes, the average daily viewing rate was $506.82 \pm 855.38$, and the average time elapsed since the videos were uploaded was $1.172 \pm 583$ days.

When we divided the quality of the video uploaders into two groups - doctors and non-doctors, it was found that 15 videos were uploaded by doctors and 35 videos were uploaded by non-doctor users. The distribution of the videos uploaded by doctors and non-doctor users according to the content is given in Figure 1 . 


\section{Cureus}

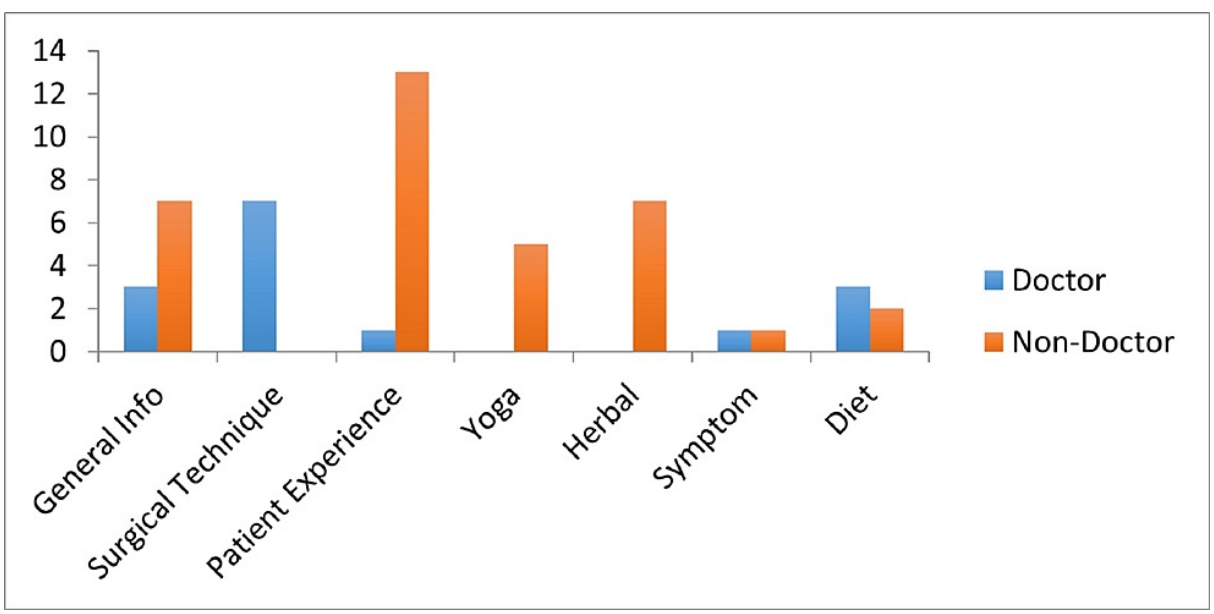

FIGURE 1: Distribution of uploaded videos by content

The mean DISCERN score given to 50 videos analyzed by two researchers was $2.81 \pm 1.3$ and the mean GQS score was $2.88 \pm 1.4$. The mean DISCERN score of the first investigator was $2.86 \pm 1.2$ and the mean GQS score was $2.94 \pm 1$. . The mean DISCERN score of the second investigator was $2.76 \pm 1.3$, and the mean GQS score was $2.82 \pm 1.3$. The average DISCERN score given by the researchers to videos uploaded by doctors was $4.43 \pm 1.27$, and the mean GQS score was $4.65 \pm 1.42$. The mean DISCERN score of non-doctor uploaders was $2.11 \pm 1.09$, and the mean GQS score was $2.12 \pm 1.26$. The scores given by the researchers to the videos according to both DISCERN and GQS are given in Table 3. 


\section{Cureus}

\begin{tabular}{|c|c|c|c|c|c|}
\hline & n (\%) & DISCERN 1 & DISCERN 2 & GQS 1 & GQS 2 \\
\hline Image & & Mean \pm SD & & & \\
\hline Real & $8(16)$ & $2.75 \pm 1.3$ & $2.62 \pm 1.4$ & $3.12 \pm 1.2$ & $3 \pm 1.3$ \\
\hline Animation & $42(74)$ & $2.88 \pm 1.2$ & $2.78 \pm 1.3$ & $2.90 \pm 1.4$ & $2.76 \pm 1.3$ \\
\hline \multicolumn{6}{|l|}{ Uploaders } \\
\hline Physician & $15(30)$ & $4.4 \pm 1.2$ & $4.46 \pm 1.3$ & $4.71 \pm 1.4$ & $4.53 \pm 1.4$ \\
\hline Hospital Channel & $4(8)$ & $2.75 \pm 1$ & $3.0 \pm 1$ & $3.25 \pm 1$ & $3 \pm 1$ \\
\hline Health Channel & $5(10)$ & $3.4 \pm 1.4$ & $3 \pm 1.1$ & $3.2 \pm 1.1$ & $3.2 \pm 1.3$ \\
\hline Patients & $13(26)$ & $2 \pm 1.5$ & $1.84 \pm 1.3$ & $1.46 \pm 1.4$ & $1.53 \pm 1.3$ \\
\hline Herbalist & $2(4)$ & $2 \pm 1.3$ & $1.5 \pm 1.2$ & $2.5 \pm 1.2$ & $2 \pm 0.8$ \\
\hline Blog Channel & $5(10)$ & $1.6 \pm 1.2$ & $1.4 \pm 1.5$ & $2 \pm 1.7$ & $2 \pm 1$ \\
\hline Yoga Channel & $6(12)$ & $1.83 \pm 1.2$ & $1.66 \pm 1.3$ & $2.16 \pm 1.1$ & $1.83 \pm 1.13$ \\
\hline \multicolumn{6}{|l|}{ Content } \\
\hline General Info & $10(20)$ & $3.5 \pm 0.8$ & $3.5 \pm 0.7$ & $3.8 \pm 0.8$ & $3.7 \pm 0.9$ \\
\hline Surgical Technique & $7(14)$ & $4.28 \pm 1.2$ & $4.22 \pm 1.3$ & $4.85 \pm 1.4$ & $4.42 \pm 1.3$ \\
\hline Patient Experience & $14(28)$ & $2.21 \pm 1.1$ & $2 \pm 1.3$ & $1.71 \pm 1.4$ & $1.71 \pm 1.3$ \\
\hline Yoga & $5(10)$ & $2 \pm 1.2$ & $1.6 \pm 1.3$ & $2.2 \pm 1.1$ & $1.8 \pm 1.4$ \\
\hline Herbal & $9(18)$ & $2 \pm 1.8$ & $1.57 \pm 1.3$ & $2.28 \pm 1.1$ & $2 \pm 1$ \\
\hline Symptom & $2(4)$ & $2.5 \pm 1.5$ & $3 \pm 1$ & $3 \pm 1$ & $3 \pm 1$ \\
\hline Diet & $3(6)$ & $2 \pm 1.2$ & $1.6 \pm 1.3$ & $2.2 \pm 1.1$ & $1.8 \pm 1.4$ \\
\hline
\end{tabular}

\section{TABLE 3: Distribution of researchers' scores on DISCERN and GQS}

GQS: Global Quality Scale

When we divided the scores given to the videos by two researchers into three groups, it was determined that $27(54 \%)$ of the 50 videos were in the misleading/poor quality group, nine (18\%) were in the medium quality group and 14 (28\%) were in the useful/quality group. The quality data of the videos according to the image type, the quality of the uploaders, and the content are shown in Table 4. 


\section{Cureus}

\begin{tabular}{|c|c|c|c|c|}
\hline & $n(\%)$ & Misleading/Harmful & Medium quality/Safe videos & Useful/Quality \\
\hline \multicolumn{5}{|l|}{ Image } \\
\hline Real & $8(16)$ & 4 & 2 & 2 \\
\hline Animation & $42(74)$ & 23 & 7 & 12 \\
\hline \multicolumn{5}{|l|}{ Uploaders } \\
\hline Doctor & $15(30)$ & 0 & 5 & 10 \\
\hline Hospital Channel & $4(8)$ & 1 & 1 & 2 \\
\hline Health Channel & $5(10)$ & 0 & 3 & 2 \\
\hline Patients & $13(26)$ & 13 & 0 & 0 \\
\hline Herbalist & $2(4)$ & 2 & 0 & 0 \\
\hline Blog Channel & $5(10)$ & 5 & 0 & 0 \\
\hline Yoga Channel & $6(12)$ & 6 & 0 & 0 \\
\hline \multicolumn{5}{|l|}{ Content } \\
\hline General Info & $10(20)$ & 0 & 5 & 5 \\
\hline Surgical Technique & $7(14)$ & 0 & 2 & 5 \\
\hline Patient Experience & $14(28)$ & 13 & 0 & 1 \\
\hline Yoga & $5(10)$ & 5 & 0 & 0 \\
\hline Herbal & $9(18)$ & 9 & 0 & 0 \\
\hline Symptom & $2(4)$ & 0 & 0 & 2 \\
\hline Diet & $3(6)$ & 0 & 2 & 1 \\
\hline
\end{tabular}

\section{TABLE 4: Distribution of videos by quality groups}

When Table 4 is examined, it is seen that all of the poor quality/misleading videos were uploaded by nondoctor users. It was determined that five of the 15 videos uploaded by the doctors were of medium quality and 10 of them contained useful and quality information. However, videos uploaded by non-doctors were found to be viewed and liked more than videos uploaded by doctors, even though they contain misleading information.

When the DISCERN and GQS scores of the two researchers were analyzed by correlation analysis, a statistically significant and strong correlation was found between researchers in terms of both DISCERN and GQS scores. However, a perfect agreement was found between the two researchers (Table 5).

\begin{tabular}{|c|c|c|c|c|}
\hline & Mean \pm SD & $p$ & $\mathbf{r}$ & Cronbach $\alpha$ \\
\hline DISCERN 1 & $2.86 \pm 1.2$ & \multirow{2}{*}{$p<0.01$} & \multirow{2}{*}{0.928} & \multirow{2}{*}{0.946} \\
\hline DISCERN 2 & $2.76 \pm 1.3$ & & & \\
\hline GQS 1 & $2.94 \pm 1.4$ & \multirow{2}{*}{$p<0.01$} & \multirow{2}{*}{0.891} & \multirow{2}{*}{0.912} \\
\hline GQS 2 & $2.82 \pm 1.3$ & & & \\
\hline
\end{tabular}

\section{TABLE 5: Correlation analysis among researchers}

GQS: Global Quality Scale 


\section{Discussion}

YouTube is the world's most-visited video-sharing platform. There are educational, entertaining, and useful videos on this platform. However, the quality of videos, especially with health content, is worried by experts. For this reason, health-related videos on YouTube have been reviewed by experts, and it has been reported that these videos are generally of bad or medium quality [8-10,13-14,17-19]. In this study, in which the quality and reliability of the videos with ovarian cyst content on YouTube were examined, it was found that 27 of the videos were of bad quality and poor quality, nine of them were of medium quality, and 14 of them were of good quality and reliability.

It was determined that the 50 videos examined were viewed $24,612,595$ times in total. In a study examining 66 videos containing hysterectomy, it was reported that the total number of views was 4.679 .118 [18]. In a study evaluating 52 videos with contraceptive implant content on YouTube, it was reported that the total number of views was 2.221.118 [19]. The differences between the number of views may vary according to the research subjects. In our study, it was determined that the videos uploaded by doctors were viewed 4.145.137 times and the videos uploaded by non-doctors were viewed 20.467.458 times. It was found that the videos uploaded by doctors were liked 44.181 times and the videos uploaded by non-doctors were liked 238.239 times. In many previous studies, it has been reported that videos uploaded by patients and other users are viewed and liked more $[9-10,13,18]$.

It was determined that $54 \%(\mathrm{n}=27)$ of the 50 videos examined in our study were of poor quality and had misleading content. Of these misleading and poor-quality videos, 13 (26\%) were uploaded by patients and their relatives, six (12\%) by yoga channels, five (10\%) by blog channels, and two (4\%) by herbalists. Similar results have been previously reported in studies that examined YouTube videos [18-19]. In the study in which hysterectomy videos were analyzed, it was reported that $51 \%$ of the videos contained misleading information and were of poor quality [18]. In another study evaluating gynecological physical examination videos, it was reported that $65 \%$ of the videos examined contained bad quality and misleading information [20].

When we examine the poor quality videos according to their content, it has been determined that all of the videos with patient experience, herbal treatment, and yoga have bad quality/misleading content. In the videos shared by the patients and their relatives, patient experiences were conveyed. These videos contain incomplete and incorrect information and were evaluated as bad/misleading by two studies. In many previous studies, it has been reported that the videos uploaded by patients have misleading/harmful content [9-14,20-21]. We think that these videos uploaded by patients should be organized with the cooperation of healthcare professionals and patients, and these videos should be shot in the attendance of experts. In addition, it was determined in our study that yoga, exercise, and herbal treatment methods also contain misleading/harmful information. These videos have been viewed more than 13 million times and have been liked 194,609 times. In similar studies, it is reported that videos with such content are viewed and liked more $[8,13-14,19,21]$.

It was determined that $18 \%(n=9)$ of the videos examined in our study were medium quality and $28 \%(n=14)$ were good quality/useful videos. Of the medium-quality videos, five were uploaded by doctors, three by health channels, and one by the hospital channel. While 10 of the good-quality videos were uploaded by doctors, two videos were uploaded by hospital channels, and two videos were uploaded by health channels. In previous similar studies, it was reported that useful videos were uploaded by doctors and health institutions [18-22]. However, in many studies, videos uploaded by doctors or healthcare organizations have also been reported to be of poor or moderate quality $[10-11,13,17]$. Researchers reported that health videos uploaded by experts on major platforms such as YouTube must undergo an absolute peer review [8-14].

In our study, when we divided the video uploaders into two groups - doctors and non-doctors, both the DISCERN and GQS scores of the videos uploaded by the doctors were statistically significantly higher than the non-doctors (for all, $\mathrm{p}<0.05$ ). In addition, a perfect agreement was found between the scores given by both DISCERN and GQS between the two researchers.

There have been some studies evaluating gynecology and obstetrics videos on YouTube. In a study evaluating 66 videos containing hysterectomy, it was reported that only $6 \%$ of the videos were of excellent quality [18]. In the study by Erdogan, 50 videos were examined and $18 \%$ of the videos were reported to be of excellent quality [11]. In another study examining 176 videos of gynecology examinations on YouTube, it was reported that $34.5 \%$ of the videos were of excellent quality [20]. In our study, $28 \%$ of the videos reviewed were found to be of excellent quality. These results show that YouTube is not a safe source for obstetrics and gynecology videos.

\section{Limitations of the study}

The primary limitation of the study is that only English videos were analyzed. It should also be noted that the video parameters on YouTube may change. The fact that we examined 50 videos in our study can be considered a limitation. However, for these 50 videos, four search terms were used and selected from 110 videos using the "relevance" and "most viewed" filtering methods. To the best of our knowledge, our study is 


\section{Conclusions}

As a result, it has been determined that videos with "ovarian cyst" content on YouTube are generally of poor quality. Bad quality videos were uploaded by non-doctors and attracted more attention than videos uploaded by doctors. We think that health-related videos should be shared by health professionals and that these videos should be peer-reviewed. In addition, in these videos shared by experts, clear information should be given instead of academic language, in a way that the viewers can understand. Finally, we believe that health professionals should share not only about diseases but also about the harms of such misleading videos.

\section{Additional Information \\ Disclosures}

Human subjects: All authors have confirmed that this study did not involve human participants or tissue. Animal subjects: All authors have confirmed that this study did not involve animal subjects or tissue. Conflicts of interest: In compliance with the ICMJE uniform disclosure form, all authors declare the following: Payment/services info: All authors have declared that no financial support was received from any organization for the submitted work. Financial relationships: All authors have declared that they have no financial relationships at present or within the previous three years with any organizations that might have an interest in the submitted work. Other relationships: All authors have declared that there are no other relationships or activities that could appear to have influenced the submitted work.

\section{References}

1. Henes M, Engler T, Taran FA, Brucker S, Rall K, Janz B, Lawrenz B: Ovarian cyst removal influences ovarian reserve dependent on histology, size and type of operation. Womens Health (Lond). 2018, 14:292-318. $10.1177 / 1745506518778992$

2. Lepine S, Jo J, Metwally M, Cheong YC: Ovarian surgery for symptom relief in women with polycystic ovary syndrome. Cochrane Database Syst Rev. 2017, 11:CD009526. 10.1002/14651858.CD009526.pub2

3. Bascietto F, Liberati M, Marrone L, et al.: Outcome of fetal ovarian cysts diagnosed on prenatal ultrasound examination: systematic review and meta-analysis. Ultrasound Obstet Gynecol. 2017, 50:20-31. 10.1002/uog.16002

4. Cooney LG, Dokras A: Depression and anxiety in polycystic ovary syndrome: etiology and treatment . Curr Psychiatry Rep. 2017, 19:83. 10.1007/s11920-017-0834-2

5. Kyaw BM, Saxena N, Posadzki P, et al.: Virtual reality for health professions education: systematic review and meta-analysis by the Digital Health Education Collaboration. J Med Internet Res. 2019, 21:e12959. $10.2196 / 12959$

6. Desai T, Shariff A, Dhingra V, Minhas D, Eure M, Kats M: Is content really king? An objective analysis of the public's response to medical videos on YouTube. PLoS One. 2013, 8:e82469. 10.1371/journal.pone.0082469

7. Kwok TM, Singla AA, Phang K, Lau AY: YouTube as a source of patient information for varicose vein treatment options. J Vasc Surg Venous Lymphat Disord. 2017, 5:238-43. 10.1016/j.jvsv.2016.10.078

8. Cakmak G, Mantoglu B: Reliability and quality of YouTube contents pertaining to pancreatic cancer . Cureus. 2021, 13:e14085. 10.7759/cureus.14085

9. Cetin A: Evaluation of YouTube video content related to the management of hypoglycemia . Cureus. 2021, 13:e12525. 10.7759/cureus.12525

10. Yurdaisik I: Analysis of the most viewed first 50 videos on YouTube about breast cancer . Biomed Res Int. 2020, 2020:2750148. 10.1155/2020/2750148

11. Erdogan G: Female genital cosmetic surgery (FGCS): evaluation of YouTube videos . J Gynecol Obstet Hum Reprod. 2021, 50:102102. 10.1016/j.jogoh.2021.102102

12. Cakmak G: Evaluation of scientific quality of YouTube video content related to umbilical hernia . Cureus. 2021, 13:e14675. 10.7759/cureus.14675

13. Kuru T, Erken HY: Evaluation of the quality and reliability of YouTube videos on rotator cuff tears . Cureus. 2020, 12:e6852. 10.7759/cureus.6852

14. Kocyigit BF, Akaltun MS, Sahin AR: YouTube as a source of information on COVID-19 and rheumatic disease link. Clin Rheumatol. 2020, 39:2049-54. 10.1007/s10067-020-05176-3

15. Singh AG, Singh S, Singh PP: YouTube for information on rheumatoid arthritis - a wakeup call? . J Rheumatol. 2012, 39:899-903. 10.3899/jrheum.111114

16. Bernard A, Langille M, Hughes S, Rose C, Leddin D, Veldhuyzen van Zanten S: A systematic review of patient inflammatory bowel disease information resources on the World Wide Web. Am J Gastroenterol. 2007, 102:2070-7. 10.1111/j.1572-0241.2007.01325.x

17. Aydin MF, Aydin MA: Quality and reliability of information available on YouTube and Google pertaining gastroesophageal reflux disease. Int J Med Inform. 2020, 137:104107. 10.1016/j.ijmedinf.2020.104107

18. Lee KN, Son GH, Park SH, Kim Y, Park ST: YouTube as a source of information and education on hysterectomy. J Korean Med Sci. 2020, 35:e196. 10.3346/jkms.2020.35.e196

19. Paul J, Boraas CM, Duvet M, Chang JC: YouTube and the single-rod contraceptive implant: a content analysis. J Fam Plann Reprod Health Care. 2017, 43:195-200. 10.1136/jfprhc-2016-101593

20. Abdulghani HM, Haque S, Ahmad T, Irshad M, Sattar K, Al-Harbi MM, Khamis N: A critical review of obstetric and gynecological physical examination videos available on YouTube. Content analysis and user engagement evaluation. Medicine (Baltimore). 2019, 98:e16459. 10.1097/MD.0000000000016459

21. Ustdal G, Guney AU: YouTube as a source of information about orthodontic clear aligners . Angle Orthod. 


\section{Cureus}

2020, 90:419-24. 10.2319/072419-491.1

22. Lee JS, Seo HS, Hong TH: YouTube as a source of patient information on gallstone disease . World J Gastroenterol. 2014, 20:4066-70. 10.3748/wig.v20.i14.4066 